\title{
Review of: "Evaluation of sample treatments in a safe and straightforward procedure for the detection of SARS-CoV-2 in saliva"
}

Hung-Sheng Shang

Potential competing interests: The author(s) declared that no potential competing interests exist.

This study provided a very useful diagnostic tool for clinical laboratory especially during the continuous COVI-19 pandemic.

The major contribution of this article provided the pre-treatment of saliva which decrease the potential exposure risk of on-site technicians still remained well clinical performance.

Here are two minor comments.

First, since collection of saliva samples by patients themselves, there might have sampling variation. Therefore, monitoring an internal control for proper sample collection should be considered as evaluation technique which we didn't found in this article.

Second, the author has mentioned RT-LAMP showed lower sensitivity than RT-PCR served as the gold standard in detecting SARS-CoV-2. We also found when Ct value $>20$, the performance of LAMP PCR decreased dramatically. The inherent lower sensitivity that may be offset by combination with other NAAT methods, we proposed the implementation of paired RT-PCR tests to counter the shortcomings of RT-LAMP tests, since false negative result e by RT-LAMP test mighty result in more large-scale transmission in SARSCoV-2 VOC situation. 